\title{
Study on the inclusive hadroproduction of $\gamma+\gamma$ at next-to-leading order
}

\author{
Rong $\mathrm{Li}^{1}$ and Jian-Xiong Wang ${ }^{2,3}$ \\ ${ }^{1}$ School of Science, Xi'an Jiaotong University, Xi'an 710049, China \\ ${ }^{2}$ Institute of High Energy Physics, Chinese Academy of Sciences, P.O. Box 918(4), Beijing 100049, China \\ ${ }^{3}$ School of Physics, University of Chinese Academy of Sciences, Beijing 100049, China
}

(Received 8 January 2019; published 13 May 2019)

\begin{abstract}
In this paper we investigate the inclusive associated production of $\gamma+\gamma$ at hadron colliders. We calculate the color-singlet subprocesses and all three color-octet subprocesses at the next-to-leading order. Seven sets of long distance matrix elements (LDMEs), which are extracted from the studies on the prompt production of $\Upsilon$ at hadron colliders, are used to give the numerical results and we find that there are three sets of LDMEs giving the unphysical results on the yield and the polarization for this process. The yield could be enhanced by several times or even two orders of magnitude compared to the color-singlet contribution and the polarization changes from longitudinal to slightly transverse or even mainly transverse in the large $p_{t}$ region. The estimated results indicate that to study the process at the Large Hadron Collider (LHC) is difficult and it may be well investigated at the future Super Proton-Proton Collider (SPPC).
\end{abstract}

DOI: 10.1103/PhysRevD.99.096010

The production and decay of heavy quarkonium have become an active area in physics since its discovery in 1974. Although there are some defects in the theoretical description, the color singlet model (CSM) had been the main tool to study the heavy quark system for about 20 years. In the early 1990s, in order to solve the $J / \psi$ surplus problem at the Tevatron [1] the nonrelativistic quantum chromodynamics (NRQCD) was proposed [2]. This new scheme also factorizes the physical process into the short distance part, which can be calculated perturbatively, and the long distance matrix elements (LDMEs), which can be extracted from matching the theoretical prediction and the experimental data or calculated by using the lattice method. The color-singlet LDMEs can also be obtained by using the calculation with the potential model [3]. The extensive studies on the NRQCD and the heavy quarkonium can be found in the reviews [4].

With the progress in calculation on the next-to-leading order (NLO) QCD correction for inclusive heavy quarkonium production many works had been done in extracting the LDMEs from fitting the theoretical predictions with experimental data [5-11]. In Ref. [12] the authors had noticed that the LDMEs of $J / \psi$ extracted from different processes cannot be consistent with each other. This may raise the doubt on the universality of the LDMEs in the

Published by the American Physical Society under the terms of the Creative Commons Attribution 4.0 International license. Further distribution of this work must maintain attribution to the author(s) and the published article's title, journal citation, and DOI. Funded by SCOAP ${ }^{3}$.
NRQCD factorization scheme. As for $\Upsilon$, the heavier bottom quark mass makes it a better one to be described by the NRQCD and the dilepton decay channel of it also provides great convenience for experimental investigation. In 2007 in the color-singlet framework the yield of inclusive production of $\gamma$ at NLO was presented in Ref. [13] and the polarization distribution was obtained in the next year [14]. The authors also estimated the results on the yield and the polarization at the partial next-to-next-toleading order [15]. Although these works were impressive progress which enhanced the $p_{t}$ distribution largely in the large $p_{t}$ region and changed the polarization from transverse to longitudinal one in the CSM, the theoretical predictions without the NLO results of color-octet channels can hardly be proper results to compare with the experimental data. Neither the leading order (LO) results of CSM or NRQCD nor the NLO results of CSM can reconcile the conflict between the theoretical results and the data on the $p_{t}$ distribution and the polarization simultaneously. In order to investigate the heavy quarkonium production at NLO in NRQCD we need not only to calculate the short distance parts at NLO but also to determine the value of corresponding LDMEs from matching the theoretical results with the experimental data. The NLO result of S-wave color-octet channel was calculated in Ref. [7]. The first theoretical calculation including the full NLO results of the color-octet channels is presented in Ref. [8]. There are some other works on the full NLO theoretical investigation of inclusive hadroproduction of $\Upsilon$ [9-11]. By using different schemes on the chosen of NRQCD scale or the fitting procedure several sets of LDMEs were obtained. Although the relative satisfactory results on the yield and the polarization 
prediction were given in the above references, there are large numerical differences among different sets of LDMEs.

In recent years, except the inclusive production of heavy quarkonium, the associated production of quarkonium has also been an attractive topic. From 1992 some authors had studied the haroproduction of quarkonium associated with a gauge boson, photon, $W^{ \pm}$or $Z^{0}$ to investigate gluon content of the proton [16] or the color octet mechanism in NRQCD at tree level [17]. The QCD NLO correction to some of these processes has been studied in Ref. [18]. The color-singlet channel of $p p \rightarrow \Upsilon+\gamma+X$ has been studied at QCD NLO [19] and the partial NNLO result has also been given [20]. The hadroproduction of $\Upsilon+\gamma$ even had been proposed in Ref. [21] as an ideal tool to investigate the transverse dynamics and polarization of gluon in proton. In our previous paper [22] we had given a full result including the NLO calculation on the color-singlet and the color-octet channel of the $J / \psi$ associated production with a photon. We had shown that several sets of LDMEs about $J / \psi$ production given different predictions on $J / \psi+\gamma$. Some of them even result in the unphysical distributions. How about the LDMEs on $\Upsilon$ which gave a good description on yield and polarization of $\boldsymbol{Y}$ inclusive production at hadron colliders? In this paper we extend our study to calculate the full results on $\Upsilon+\gamma$ at NLO.

According to the NRQCD factorization scheme, the differential cross section for this process can be expressed as,

$$
\begin{aligned}
& \sigma(p+p \rightarrow \Upsilon+\gamma+X) \\
& \quad=\sum_{i, j, n} \int d x_{1} d x_{2} G_{p}^{i}\left(x_{1}\right) G_{p}^{j}\left(x_{2}\right) \hat{\sigma}\left(i j \rightarrow(Q \bar{Q})_{n}+\gamma+X\right)\left\langle O_{n}^{\Upsilon}\right\rangle .
\end{aligned}
$$

The above equation indicates that the cross section of this process is the convolution of the parton distribution function $G_{p}^{i(j)}$ and the parton level short distance coefficients $\hat{\sigma}$. The $\left\langle O_{n}^{\Upsilon}\right\rangle$ is the LDME of corresponding subprocesses. The parton level subprocesses are,

$$
\begin{gathered}
g+g \rightarrow Q \bar{Q}\left[{ }^{3} S_{1}^{1},{ }^{1} S_{0}^{8},{ }^{3} S_{1}^{8},{ }^{3} P_{J}^{8}\right]+\gamma, \\
q+\bar{q} \rightarrow Q \bar{Q}\left[{ }^{1} S_{0}^{8},{ }^{3} S_{1}^{8},{ }^{3} P_{J}^{8}\right]+\gamma, \\
g+g \rightarrow Q \bar{Q}\left[{ }^{3} S_{1}^{1},{ }^{1} S_{0}^{8},{ }^{3} S_{1}^{8},{ }^{3} P_{J}^{8}\right]+\gamma+g . \\
q+\bar{q} \rightarrow Q \bar{Q}\left[{ }^{3} S_{1}^{1},{ }^{1} S_{0}^{8},{ }^{3} S_{1}^{8},{ }^{3} P_{J}^{8}\right]+\gamma+g . \\
g+q(\bar{q}) \rightarrow Q \bar{Q}\left[{ }^{3} S_{1}^{1},{ }^{1} S_{0}^{8},{ }^{3} S_{1}^{8},{ }^{3} P_{J}^{8}\right]+\gamma+q(\bar{q}) .
\end{gathered}
$$

The ${ }^{2 S+1} L_{J}^{1,8}$ is the spectroscopic notation, where $S$ is the total spin of the intermediate state, $L$ is the orbital angular momentum, $J$ is the total angular momentum, and 1 or 8 presents the color state. For the description of $\Upsilon$ polarization we adopt the usual definition in helicity frame as

$$
\alpha\left(p_{t}\right)=\frac{\mathrm{d} \sigma_{11} / \mathrm{d} p_{t}-\mathrm{d} \sigma_{00} / \mathrm{d} p_{t}}{\mathrm{~d} \sigma_{11} / \mathrm{d} p_{t}+\mathrm{d} \sigma_{00} / \mathrm{d} p_{t}}
$$

The $d \sigma_{i j}$ are the differential production density matrix elements and $i(j)=1,0$ mean the transverse and longitudinal polarization of $\Upsilon$.

There is infrared divergence in the subprocesses (6) when the photon is collinear with the light quark. This infrared divergence should cancel with the quantum electrodynamic correction of $g+g \rightarrow \Upsilon+g$. Because we want to observe the photon we use the isolated condition in Ref. [23] to isolate the photon from the quark jet in these subprocesses. The isolated condition is

$$
p_{t}^{i} \leq p_{t}^{\gamma} \frac{1-\cos R_{\gamma_{i}}}{1-\cos \delta_{0}} \quad \text { for } R_{\gamma_{i}}<\delta_{0}
$$

The definitions of the $p_{t}^{i}, p_{t}^{\gamma}, \cos R_{\gamma_{i}}$ and the $\delta_{0}$ can be found in Ref. [23]. Here we set $\delta_{0}=0.7$. The newly infrared divergence appearing in the calculation of $\mathrm{P}$-wave processes at NLO, which come from the long distance evolution of the heavy $Q \bar{Q}$ pair into a physical quarkonium state, will be factorized by $\mu_{\Lambda}$ and absorbed into the redefinition of the long distance matrix elements, which we had used in our previous papers [24]. We use the Feynman diagram calculation (FDC) package [25] to generate the analytic results and output the FORTRAN code for numerical evaluation.

Let us talk about the choice of parameters used in the numerical calculation. In the calculation, the bottom quark mass is set as $4.73 \mathrm{GeV}$ and will vary from 4.63 to 4.83 to estimate the related uncertainties. The factorization and the renormalization scales are set as $\mu_{r}=\mu_{f}=\mu_{0}=$ $\sqrt{\left(2 m_{b}\right)^{2}+p_{t}^{2}}$ and will vary from $\mu_{0} / 2$ to $2 \mu_{0}$ to estimate the uncertainties. The CTEQ6L amd CTEQ6M PDFs are used in the calculation of the LO and NLO convolutions and the $\alpha_{s}$ running in these PDFs are used to calculate the cross sections of the subprocesses. The center of mass energy and cut condition for the $\Upsilon$ or the final photon are set as $\sqrt{s}=7,8,14 \mathrm{TeV},\left|y_{\Upsilon, \gamma}\right| \leq 3,\left|\eta_{\gamma}\right| \leq 1.45$ and $p_{t}^{\gamma}>1.5,3,5,15 \mathrm{GeV}$. The fine structure constant for the electromagnetic coupling is chosen as $\frac{1}{128}$. The different choice of the parameters are marked in the figures.

The extraction of the LDMEs in NRQCD is the key point to give the rational theoretical predictions. As for the LDMEs for the production of $\Upsilon$ there are many sets of them extracted by two groups form Peking University (PKU) $[8,10]$ and the Institute of High Energy Physics [9,11]. In Ref. [8] the authors gave the three LDMEs at NLO for the first time. But they included the feed-down contribution from ${ }^{3} S_{1}^{[8]}$ channel of P-wave bottomonium in the corresponding CO LDMEs of $\Upsilon$. Therefore, we do not use the LDMEs in Ref. [8] in our numerical calculation. The authors in Ref. [9] extracted the LDMEs related to the production of $\Upsilon$ individually by matching their theoretical 
TABLE I. The NRQCD LDMEs $\left\langle O^{\mathrm{Y}}(n)\right\rangle$ extracted by two groups at the NLO with $\left\langle O^{\mathrm{r}}\left({ }^{3} S_{1}^{1}\right)\right\rangle=9.28 \mathrm{GeV}^{3}$. The other three color-octet LDMEs are listed as follows. (LDMEs in units of $10^{-2} \mathrm{GeV}^{3}, P_{t}^{\mathrm{r}}$ and $\mu_{\Lambda}$ in units of $\mathrm{GeV}$.)

\begin{tabular}{lcrccc}
\hline \hline & $O\left({ }^{1} S_{0}^{8}\right)$ & $O\left({ }^{3} S_{1}^{8}\right)$ & $O\left({ }^{3} P_{0}^{8}\right) / m_{b}^{2}$ & $P_{t}^{\mathrm{r}}>$ & $\mu_{\Lambda}$ \\
\hline Han [10] & 0.017 & 2.97 & 3.83 & 15 & $m_{b}$ \\
Han1 [10] & 0 & 3.04 & 3.61 & 15 & $m_{b}$ \\
Han2 [10] & 13.7 & 1.17 & 0 & 15 & $m_{b}$ \\
Gong [9] & 11.15 & -0.41 & -0.67 & 8 & $m_{b} v$ \\
Feng1 [11] & 13.6 & 0.61 & -0.93 & 8 & $m_{b} v$ \\
Feng2 [11] & 10.1 & 0.73 & -0.23 & 8 & $m_{b} v$ \\
Feng3 [11] & 11.6 & 0.47 & -0.49 & 8 & $m_{b}$ \\
\hline \hline
\end{tabular}

prediction at NLO with the yield and the polarization at Tevatron and LHC. Thereafter, they updated their analysis with three different schemes by including the newly measured parameters, the mass of $\Upsilon(3 S)$ and the fraction for $\chi_{b J}(3 P) \rightarrow \Upsilon(3 S)$ [11]. The PKU group also extracted the individual LDMEs in the first version of Ref. [10] by matching the yield and the polarization data. In the second version of Ref. [10] they decomposed the contribution of P-wave color-octet subprocesses into the linear combination of the two S-wave subprocesses. Therefore, just as in the case of $J / \psi$ production [6] they extracted two linear combination of the three LDMEs as

$$
\begin{aligned}
& M_{0, r_{0}}^{\mathrm{r}}=\left\langle O^{\mathrm{\Upsilon}}\left({ }^{1} S_{0}^{8}\right)\right\rangle+\frac{r_{0}}{m_{b}^{2}}\left\langle O^{\Upsilon}\left({ }^{3} P_{0}^{8}\right)\right\rangle, \\
& M_{1, r_{1}}^{\mathrm{\gamma}}=\left\langle O^{\Upsilon}\left({ }^{3} S_{1}^{8}\right)\right\rangle+\frac{r_{1}}{m_{b}^{2}}\left\langle O^{\Upsilon}\left({ }^{P} P_{0}^{8}\right)\right\rangle,
\end{aligned}
$$

where $r_{0}=3.8, r_{1}=-0.52, M_{0, r_{0}}^{\mathrm{r}}=13.70 \times 10^{-2} \mathrm{GeV}^{3}$ and $M_{1, r_{1}}^{\mathrm{r}}=1.17 \times 10^{-2} \mathrm{GeV}^{3}$. Here we use the same scheme as in our previous paper on $J / \psi+\gamma$. By requiring the LDMEs to be positive we obtain two sets of LDMEs referring to as "Han Extension" (Han1 and Han2) in the following parts. The seven sets of LDMEs and related parameters, the $\mu_{\Lambda}$ and the cuts on the transverse momentum of $\Upsilon\left(P_{t}^{\Upsilon}\right)$, are listed in Table I.

In Fig. 1 we show some features of the subprocesses. The ratios between the three subprocesses are plotted in the upper parts of the Fig. 1. We can see that with the increase of transverse momentum the cross sections for the ${ }^{3} S_{1}^{8}$ and ${ }^{3} P_{J}^{8}$ subprocesses at the parton level become much larger than that of the ${ }^{1} S_{0}^{8}$ subprocess and there is no linear correlation among them, just as the associated production of $J / \psi+\gamma$. Because the ${ }^{1} S_{0}^{8}$ subprocess is unpolarized we just show the polarization of the other two subprocesses in the lower part of Fig. 1. Both of the previous two subprocesses show the transverse polarization in almost the whole $P_{t}$ region. This two figures together with the

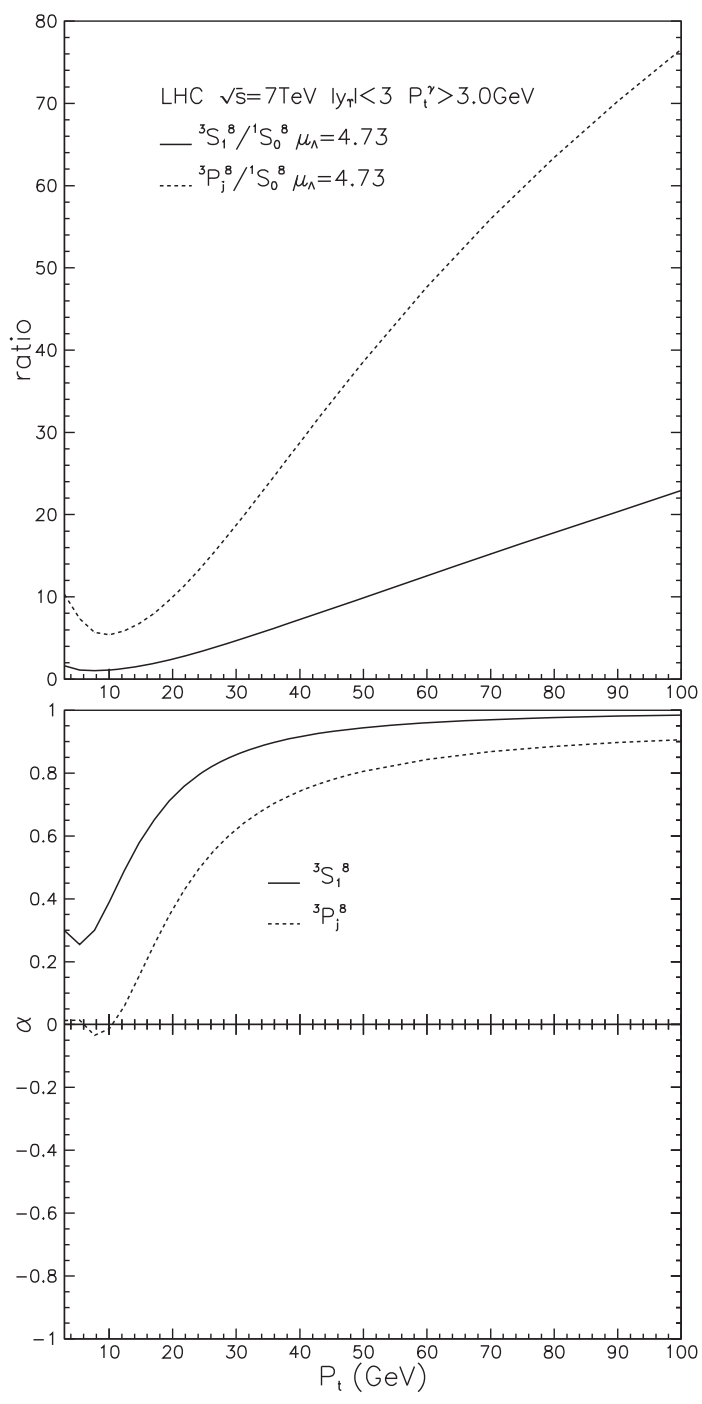

FIG. 1. The ratio of $d \sigma\left({ }^{3} P_{J}^{8}\right) / d \sigma\left({ }^{1} S_{0}^{8}\right)$ and $d \sigma\left({ }^{3} S_{1}^{8}\right) / d \sigma\left({ }^{1} S_{0}^{8}\right)$ as functions of $P_{t}$ and the polarization for the subprocesses.

choice of the LDMEs will give qualitative hints on the polarization of the final $\Upsilon$.

Because the contribution from ${ }^{3} S_{1}^{8}$ and ${ }^{3} P_{J}^{8}$ subprocesses is dominant, the numerical results with the Han set are almost the same as that of the Han1. We just show the results of two extension cases. There is similar situation between the Gong and the Feng 1 sets. The sets Feng 1 and Feng3 give the similar trend on the yield and the polarization, which become unphysical in the large $p_{t}$ region. Therefore, we just plot the numerical results with the LDMEs sets Han extension1, Han extension2, Feng1, and Feng2 in the following parts in our paper.

We plot three sets of results in three columns in Fig. 2. In the first column we investigate the dependence on the $p_{t}^{\gamma}$ cuts for $p_{t}$ distribution of the yield and polarization. We choose three $p_{t}^{\gamma}$ cuts and plot the corresponding curves. Comparing to the CSM results at NLO even at the large $p_{t}$ region (about $100 \mathrm{GeV}$ ) the cross section and the 

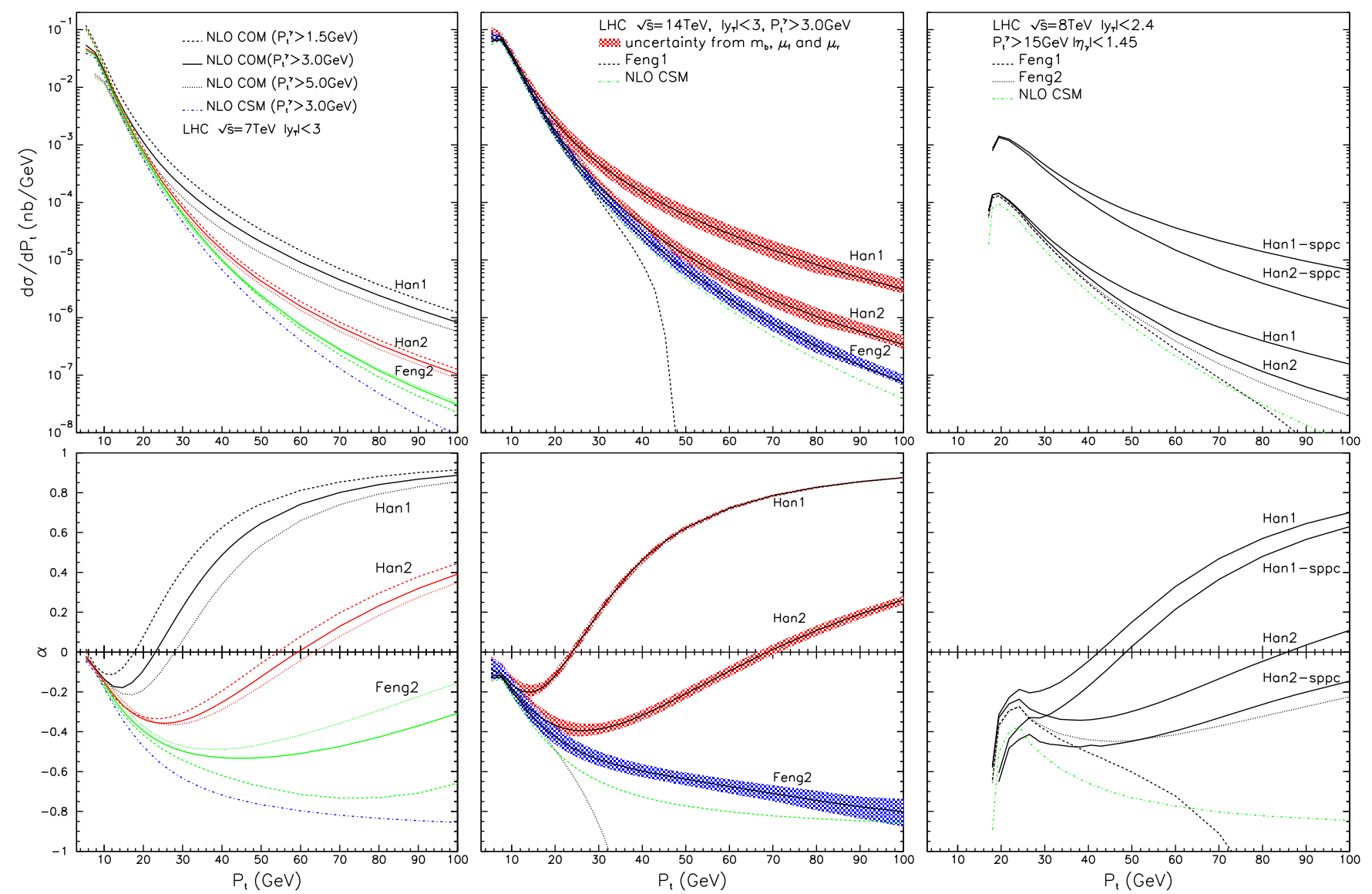

FIG. 2. The $p_{t}(\Upsilon)$ distributions for $\Upsilon+\gamma$ production (upper parts) and polarization (lower parts) with different conditions. Figures in the same column are of the same conditions and line types. The shaded band in the second column represent the uncertainty from variation of $\mu_{f}$ and $\mu_{r}$ and $m_{b}$. The third column shows results with $p_{t}^{\gamma}>15 \mathrm{GeV}$ and different LDME sets.

polarization do not have a manifestly convergence behavior in the $p_{t}$ region being studied. This is due to the infrared divergence related to the photon which is handled by using the isolated condition. They almost have a parallel shift with different $p_{t}^{\gamma}$ cuts.

The second column in Fig. 2 shows the results at $\sqrt{s}=14 \mathrm{GeV}$. The shaded band presents the dependence of physical observables on three parameters, bottom quark mass $m_{b}$, renormalization scale $\mu_{r}$ and the factorization scale $\mu_{f}$. The numerical results show the similar feature as that in the $J / \psi$ case [22]. At first by including the contribution of color-octet processes, the cross section for the inclusive hadroproduction of $\Upsilon+\gamma$ is about 2 or 70 times larger than that of the CSM result at NLO with three sets of LDMEs which can give physical results. The polarization of $\Upsilon$ changes from mainly longitudinal in CSM to slightly longitudinal, slightly transverse and even mainly transverse at NLO with three sets of LDMEs. The uncertainty of production rate becomes larger with the increase of $p_{t}$ and at the same time the polarization parameter exhibit more sophisticated feature. The polarization parameter obtained by using the Han extension sets of LDMEs converges with the increase of $p_{t}$ and the one with the Feng2 set of LDMEs does not. Considering the values of the LDMEs we can infer that the uncertainty mainly comes from the ${ }^{1} S_{0}^{8}$ channel. The results with the Feng1 set of LDMEs are also plotted in the figures, which give negative cross section in the $p_{t}$ distribution at about $p_{t}=48 \mathrm{GeV}$ and result in a polarization parameter lower than -1 when $p_{t}>32 \mathrm{GeV}$.

Just as in the case of $J / \psi+\gamma$ the associated production of $\gamma+\gamma$ provides an opportunity to distinguish or to assess the different sets of LDMEs obtained with different schemes on $\Upsilon$ production. We calculate the theoretical predictions for the yield and the polarization of $\Upsilon$ in this process with the $\sqrt{s}=8 \mathrm{GeV}$. The LHC has $23 \mathrm{fb}^{-1}$ integrated luminosity at $\sqrt{s}=8 \mathrm{GeV}$. We choose $p_{t}^{\gamma}>$ $15 \mathrm{GeV}$ to suppress the background and $\eta_{\gamma}<1.45$ for photon reconstruction efficiency consideration. The numerical results show that in the large $p_{t}$ region the differential cross section which, including the COM contribution, is about 2 to 20 times larger than the CSM result. The ratio is smaller than that of the $J / \psi+\gamma$. The Feng 1 set of LDMEs also gives the unphysical predictions on the $p_{t}$ yield and the 
polarization. The photon reconstruction efficiency under the above pseudorapidity and $p_{t}$ cut condition is 0.7 [26]. The branch ratio of $\Upsilon$ dilepton decay channel is $\operatorname{Br}\left(\Upsilon \rightarrow \mu^{+} \mu^{-}\right.$and $\left.e^{+} e^{-}\right)=0.05$. Therefore we can expect there are $0.5-5$ events at $p_{t}^{\mathrm{r}}=100 \mathrm{GeV}$ or 9-30 events at $p_{t}^{\mathrm{r}}=60 \mathrm{GeV}$ with $\sqrt{s}=8 \mathrm{TeV}$. If the center of mass energy is up to $100 \mathrm{TeV}$ at the Super Proton-Proton Collider (SPPC) and the other conditions are unchanged, we can see from the third column that the differential cross section could be 40 times larger than that with $\sqrt{s}=8 \mathrm{TeV}$ and the polarization of $\Upsilon$ changes from longitudinal to transverse more rapidly. By using the previous reconstruction efficiency and the branch ratio we can obtain 32-161 (Han1) or 322-782 (Han2) event numbers, respectively. It means this process should be well studied at SPPC.

In summary, we investigate the inclusive production of $\gamma+\gamma$ at the hadron collider. By including the three different color-octet subprocesses we give complete theoretical predictions on the yield and the polarization at QCD NLO within the NRQCD framework. Comparing to our previous work [22] the cross section for this process is enhanced several times or even two orders in the large $p_{t}$ region. The inclusion of color-octet subprocesses at NLO changes the polarization from longitudinal to transverse. Although the scale chosen and the variation of the bottom quark mass can bring the uncertainties to some extent, the main uncertainty of the theoretical prediction comes from the different LDMEs set. We investigate seven sets of LDMEs and only four of them, Han, Han extension1, Han extension2, and Feng 2 can give the physical predictions on the yield and the polarization in the $p_{t}$ region that we studied. From Fig. 1 it can be seen that the P-wave coloroctet subprocess gives the dominate contribution in the large $p_{t}$ region. Therefore, we infer that any set of LDMEs with negative $O\left({ }^{3} P_{0}^{8}\right)$ will result in the negative prediction on the yield at sufficiently large $p_{t}$ region and the Feng2 set may meet this problem with the increase of $p_{t}$. The estimate with LHC integrated luminosity at $\sqrt{s}=8 \mathrm{TeV}$ indicates that it is not optimistic to investigate this process at the LHC. The result with $\sqrt{s}=100 \mathrm{TeV}$ shows us this process can be studied in the next generation hadron colliders, such as the SPPC.

We acknowledge the support from the National Natural Science Foundation of China under Grants No. 11105152, No. 11375137 and No. U1832160, the Natural Science Foundation of Shaanxi Province under Grant No. 2015JQ1003, the Fundamental Research Funds for the Central Universities, and the Key Research Program of Frontier Sciences, CAS, Grant No. Y7292610K1.
[1] F. Abe et al. (CDF Collaboration), Phys. Rev. Lett. 69, 3704 (1992).

[2] G. T. Bodwin, E. Braaten, and G. P. Lepage, Phys. Rev. D 51, 1125 (1995); 55, 5853 (1997).

[3] E. J. Eichten and C. Quigg, Phys. Rev. D 52, 1726 (1995).

[4] A. Andronic et al., Eur. Phys. J. C 76, 107 (2016); G. T. Bodwin, E. Braaten, E. Eichten, S. L. Olsen, T. K. Pedlar, and J. Russ, arXiv:1307.7425; N. Brambilla et al., Eur. Phys. J. C 71, 1534 (2011); N. Brambilla et al. (Quarkonium Working Group), arXiv:hep-ph/0412158; J. P. Lansberg, Int. J. Mod. Phys. A 21, 3857 (2006).

[5] Y. Q. Ma, K. Wang, and K. T. Chao, Phys. Rev. Lett. 106, 042002 (2011); M. Butenschoen and B. A. Kniehl, Phys. Rev. Lett. 106, 022003 (2011); Y. Q. Ma, K. Wang, and K. T. Chao, Phys. Rev. D 84, 114001 (2011); M. Butenschoen and B. A. Kniehl, Phys. Rev. D 84, 051501 (2011); Phys. Rev. Lett. 107, 232001 (2011); K.-T. Chao, Y.-Q. Ma, H.-S. Shao, K. Wang, and Y.-J. Zhang, Phys. Rev. Lett. 108, 242004 (2012); A. K. Likhoded, A. V. Luchinsky, and S. V. Poslavsky, Phys. Rev. D 86, 074027 (2012); B. Gong, L.-P. Wan, J.-X. Wang, and H.-F. Zhang, Phys. Rev. Lett. 110, 042002 (2013); A. K. Likhoded, A. V. Luchinsky, and S. V. Poslavsky, Phys. Rev. D 90, 074021 (2014).

[6] Y.-Q. Ma, K. Wang, and K.-T. Chao, Phys. Rev. D 83, 111503 (2011).
[7] B. Gong, J. X. Wang, and H. F. Zhang, Phys. Rev. D 83, 114021 (2011).

[8] K. Wang, Y. Q. Ma, and K. T. Chao, Phys. Rev. D 85, 114003 (2012).

[9] B. Gong, L.-P. Wan, J.-X. Wang, and H.-F. Zhang, Phys. Rev. Lett. 112, 032001 (2014).

[10] H. Han, Y. Q. Ma, C. Meng, H. S. Shao, Y. J. Zhang, and K. T. Chao, Phys. Rev. D 94, 014028 (2016).

[11] Y. Feng, B. Gong, L. P. Wan, and J. X. Wang, Chin. Phys. C 39, 123102 (2015).

[12] M. Butenschoen and B. A. Kniehl, Phys. Rev. Lett. 108, 172002 (2012).

[13] J. M. Campbell, F. Maltoni, and F. Tramontano, Phys. Rev. Lett. 98, 252002 (2007).

[14] B. Gong and J. X. Wang, Phys. Rev. D 78, 074011 (2008).

[15] P. Artoisenet, J. M. Campbell, J. P. Lansberg, F. Maltoni, and F. Tramontano, Phys. Rev. Lett. 101, 152001 (2008).

[16] M. Drees and C. S. Kim, Z. Phys. C 53, 673 (1992); M. A. Doncheski and C.S. Kim, Phys. Rev. D 49, 4463 (1994).

[17] E. Mirkes and C. S. Kim, Phys. Rev. D 51, 3340 (1995); Phys. Lett. B 346, 124 (1995); D. P. Roy and K. Sridhar, Phys. Lett. B 341, 413 (1995); C. S. Kim, J. Lee, and H. S. Song, Phys. Rev. D 55, 5429 (1997); P. Mathews, K. Sridhar, and R. Basu, Phys. Rev. D 60, 014009 (1999); B. A. Kniehl, C. P. Palisoc, and L. Zwirner, Phys. Rev. D 66, 114002 (2002); J. P. Lansberg and C. Lorce, Phys. Lett. B 726, 218 (2013). 
[18] G. Li, S. Mao, R.-Y. Zhang, and W.-G. Ma, Phys. Rev. D 83, 014001 (2011); S. Mao, M. Wen-Gan, L. Gang, Z. Ren-You, and G. Lei, J. High Energy Phys. 02 (2011) 071; 12 (2012) 010; B. Gong, J.-P. Lansberg, C. Lorce, and J. Wang, J. High Energy Phys. 03 (2013) 115.

[19] R. Li and J.-X. Wang, Phys. Lett. B 672, 51 (2009).

[20] J. P. Lansberg, Phys. Lett. B 679, 340 (2009).

[21] W. J. den Dunnen, J. P. Lansberg, C. Pisano, and M. Schlegel, Phys. Rev. Lett. 112, 212001 (2014).
[22] R. Li and J. X. Wang, Phys. Rev. D 89, 114018 (2014).

[23] S. Frixione, Phys. Lett. B 429, 369 (1998).

[24] J.-X. Wang and H.-F. Zhang, Phys. Rev. D 86, 074012 (2012).

[25] J.-X. Wang, Nucl. Instrum. Methods Phys. Res., Sect. A 534, 241 (2004); The new homepage for FDC is http:// www1.ihep.ac.cn/wjx/index.html.

[26] CMS Collaboration, CERN Report No. CMS-DP-2013010, 2013. 